\title{
ASYMPTOTIC ESTIMATES AND EXPONENTIAL STABILITY FOR HIGHER-ORDER MONOTONE DIFFERENCE EQUATIONS
}

EDUARDO LIZ AND MIHÁLY PITUK

Received 21 May 2004

Asymptotic estimates are established for higher-order scalar difference equations and inequalities the right-hand sides of which generate a monotone system with respect to the discrete exponential ordering. It is shown that in some cases the exponential estimates can be replaced with a more precise limit relation. As corollaries, a generalization of discrete Halanay-type inequalities and explicit sufficient conditions for the global exponential stability of the zero solution are given.

\section{Introduction}

Consider the higher-order scalar difference equation

$$
x_{n+1}=f\left(x_{n}, x_{n-1}, \ldots, x_{n-k}\right), \quad n \in \mathbb{N}=\{0,1,2, \ldots\},
$$

where $k$ is a positive integer and $f: \mathbb{R}^{k+1} \rightarrow \mathbb{R}$. With (1.1), we can associate the discrete dynamical system $\left(T^{n}\right)_{n \geq 0}$ on $\mathbb{R}^{k+1}$, where $T: \mathbb{R}^{k+1} \rightarrow \mathbb{R}^{k+1}$ is defined by

$$
T(x)=\left(f(x), x_{0}, x_{1}, \ldots, x_{k-1}\right), \quad x=\left(x_{0}, x_{1}, \ldots, x_{k}\right) \in \mathbb{R}^{k+1} .
$$

As usual, $T^{n}$ denotes the $n$th iterate of $T$ for $n \geq 1$ and $T^{0}=I$, the identity on $\mathbb{R}^{k+1}$. It follows by easy induction on $n$ that if $\left(x_{n}\right)_{n \geq-k}$ is a solution of (1.1), then

$$
\left(x_{n}, x_{n-1}, \ldots, x_{n-k}\right)=T^{n}\left(x_{0}, x_{-1}, \ldots, x_{-k}\right), \quad n \geq 0 .
$$

Therefore, the dynamical system $\left(T^{n}\right)_{n \geq 0}$ contains all information about the behavior of the solutions of (1.1).

In a recent paper [7], motivated by earlier results for delay differential equations due to Smith and Thieme [13] (see also [12, Chapter 6]), Krause and the second author have introduced the discrete exponential ordering on $\mathbb{R}^{k+1}$, the partial ordering induced by the convex closed cone

$$
C_{\mu}=\left\{x=\left(x_{0}, x_{1}, \ldots, x_{k}\right) \in \mathbb{R}^{k+1} \mid x_{k} \geq 0, x_{i} \geq \mu x_{i+1}, i=0,1, \ldots, k-1\right\},
$$


where $\mu \geq 0$ is a parameter. In [7], it has been shown that $T$ is monotone (order preserving) under appropriate conditions on $f$. As a consequence of monotonicity, necessary and sufficient conditions have been given for the boundedness of all solutions and for the local and global stability of an equilibrium of (1.1) (see [7, Section 4]).

In this paper, we give further consequences of the monotonicity of $T$ for (1.1) and for the corresponding difference inequality

$$
y_{n+1} \leq f\left(y_{n}, y_{n-1}, \ldots, y_{n-k}\right), \quad n \geq 0,
$$

under the additional assumption that the nonlinearity $f$ is positively homogeneous (of degree one) on the generating cone $C_{\mu}$, that is,

$$
f(\lambda x)=\lambda f(x) \text { for } \lambda \geq 0, x \in C_{\mu} .
$$

An example of (1.1) with property (1.6) is the max type difference equation

$$
x_{n+1}=\sum_{i=0}^{k} K_{i} x_{n-i}+b \max \left\{x_{n}, x_{n-1}, \ldots, x_{n-r}\right\},
$$

where $k$ and $r$ are positive integers and the coefficients $K_{i}$ and $b$ are constants. For other examples of higher-order difference equations with a positively homogeneous right-hand side, see, for example, [6].

Using the monotonicity of $T$ and a simple comparison theorem, we give upper exponential estimates for the solutions of (1.5) in terms of the largest positive root of the characteristic equation

$$
\lambda^{k+1}=f\left(\lambda^{k}, \lambda^{k-1}, \ldots, 1\right)
$$

As a corollary for the difference inequality

$$
y_{n+1} \leq \sum_{i=0}^{k} K_{i} y_{n-i}+b \max \left\{y_{n}, y_{n-1}, \ldots, y_{n-r}\right\} \text {, }
$$

we obtain a generalization of earlier results of Ferreiro and the first author [8] on discrete Halanay-type inequalities (see Theorems 1.1 and 3.1). For other related results, see, for example, $[1,9,10]$.

Further, we will show that a mild strengthening of the monotonicity condition in [7] implies that the map $T$ is eventually strongly monotone. As a consequence, a nonlinear version of the Perron-Frobenius theorem [3] applies and we obtain an asymptotic representation of the solutions of (1.1) starting from $C_{\mu}$ (see Theorems 1.2 and 3.7). For a similar result, using the standard ordering in $\mathbb{R}^{k+1}(\mu=0)$, see [6].

Finally, we establish an asymptotic exponential estimate for the growth of the solutions of the equation

$$
x_{n+1}=\sum_{i=0}^{k} K_{i} x_{n-i}+g\left(n, x_{n}, x_{n-1}, \ldots, x_{n-r}\right),
$$


under the assumption that its linear part

$$
y_{n+1}=\sum_{i=0}^{k} K_{i} y_{n-i}
$$

generates a monotone system and the growth of the nonlinearity $g: \mathbb{N} \times \mathbb{R}^{r+1} \rightarrow \mathbb{R}$ is controlled by a positively homogeneous function which is nondecreasing in each of its variables (see Theorems 1.3 and 3.10). As a corollary, we obtain explicit sufficient conditions for the global exponential stability of the zero solution of (1.10) (see Theorems 1.4 and 3.11).

The following four theorems give a flavor of our more general results presented in Section 3. Without loss of generality, we assume that in all Theorems 1.1, 1.2, 1.3, and 1.4 below, $k \geq r$. The first theorem offers an upper estimate for the solutions of inequality (1.9).

Theorem 1.1. Suppose that $b>0$ and there exists $\mu>0$ such that

$$
\mu+\sum_{i=1}^{k} K_{i}^{-} \mu^{-i} \leq K_{0},
$$

where $K_{i}^{-}=\max \left\{0,-K_{i}\right\}$. Then, for every solution $\left(y_{n}\right)_{n \geq-k}$ of (1.9) there exists a positive constant $M=M\left(y_{0}, y_{-1}, \ldots, y_{-k}\right)$ such that

$$
y_{n} \leq M \lambda_{0}^{n}, \quad n \geq-k,
$$

where $\lambda_{0}$ is the unique root of the equation

$$
\lambda^{k+1}=\sum_{i=0}^{k} K_{i} \lambda^{k-i}+b \max \left\{\lambda^{k}, \lambda^{k-1}, \ldots, \lambda^{k-r}\right\}
$$

in the interval $(\mu, \infty)$.

The next result shows in case of (1.7) the exponential estimate (1.13) of Theorem 1.1 is sharp.

Theorem 1.2. Suppose that $b>0$ and (1.12) holds with a strict inequality for some $\mu>0$. Then, for every solution $\left(x_{n}\right)_{n \geq-k}$ of (1.7) with initial data $\left(x_{0}, x_{-1}, \ldots, x_{-k}\right) \in C_{\mu} \backslash\{0\}$, there exists a positive constant $L=L\left(x_{0}, x_{-1}, \ldots, x_{-k}\right)$ such that

$$
\lambda_{0}^{-n} x_{n} \longrightarrow L \text { as } n \longrightarrow \infty
$$

where $\lambda_{0}$ has the meaning from Theorem 1.1.

The following theorem provides an estimate for the growth of the solutions of (1.10). Theorem 1.3. Suppose that there exist $b>0$ and $\mu>0$ such that (1.12) and

$$
\left|g\left(n, x_{0}, x_{1}, \ldots, x_{r}\right)\right| \leq b \max \left\{\left|x_{0}\right|,\left|x_{1}\right|, \ldots,\left|x_{r}\right|\right\}, \quad n \geq 0, x \in \mathbb{R}^{r+1}
$$


hold. Then, for every solution $\left(x_{n}\right)_{n \geq-k}$ of (1.10) there exists a positive constant $M=M\left(x_{0}\right.$, $\left.x_{-1}, \ldots, x_{-k}\right)$ such that

$$
\left|x_{n}\right| \leq M \lambda_{0}^{n}, \quad n \geq-k
$$

where $\lambda_{0}$ has the meaning from Theorem 1.1.

The existence and uniqueness of the solution $\lambda_{0}$ of $(1.14)$ in $(\mu, \infty)$ is a part of the conclusions of Theorems 1.1, 1.2, and 1.3. This $\lambda_{0}$ is a root of either

$$
\lambda^{k+1}=\sum_{i=0}^{k} K_{i} \lambda^{k-i}+b \lambda^{k}
$$

or

$$
\lambda^{k+1}=\sum_{i=0}^{k} K_{i} \lambda^{k-i}+b \lambda^{k-r},
$$

depending on whether $\lambda_{0} \geq 1$ or $\lambda_{0}<1$. It will be shown (see Corollary 2.7) that $\lambda_{0}<1$ if and only if, in addition to the hypotheses of Theorem 1.1, $\mu<1$ and

$$
\sum_{i=0}^{k} K_{i}+b<1 .
$$

As a consequence of Theorem 1.3, we have the following criterion for the global exponential stability of the zero solution of (1.10).

Theorem 1.4. Suppose that there exist $b>0$ and $\mu \in(0,1)$ such that (1.12), (1.16), and (1.20) hold. Then, the zero solution of (1.10) is globally exponentially stable.

For the proofs of Theorems 1.1, 1.2, 1.3, and 1.4, see Remarks 3.4, 3.9 and, 3.12.

In the special case $K_{0} \geq 0, K_{i}=0$ for $i=1,2, \ldots, k$ and $0<b<1-K_{0}$, the conclusion of Theorem 1.1, a discrete analogue of Halanay's inequality, was obtained by Ferreiro and the first author (see [8, Theorem 1]). The same remark holds for Theorem 1.4 (see [8, Theorem 2]).

Under the hypotheses of Theorem 1.4, the global asymptotic stability of the zero solution of (1.10) was established by the second author using a different approach (see [11, Corollary 2 and Remark 2]).

The paper is organized as follows. In Section 2, we discuss the monotonicity properties of the map $T$ defined by (1.2). The main results on the behavior of the solutions of the above higher-order difference equations and inequalities are given in Section 3.

\section{Monotonicity}

Recall the definition of the discrete exponential ordering from [7]. For every $\mu \geq 0$, the convex closed cone $C_{\mu}$ defined by (1.4) has nonempty interior int $C_{\mu}$ given by

$$
\operatorname{int} C_{\mu}=\left\{x=\left(x_{0}, x_{1}, \ldots, x_{k}\right) \in \mathbb{R}^{k+1} \mid x_{k}>0, x_{i}>\mu x_{i+1}, i=0,1, \ldots, k-1\right\} \text {. }
$$


As a cone in $\mathbb{R}^{k+1}$, each $C_{\mu}$ induces a partial order $\leq_{\mu}$ on $\mathbb{R}^{k+1}$ by $x \leq_{\mu} y$ if and only if $y-x \in C_{\mu}$. We write $x<_{\mu} y$ if $x \leq_{\mu} y$ and $x \neq y$. The strong ordering $\ll_{\mu}$ is defined by $x \ll_{\mu} y$ if and only if $y-x \in \operatorname{int} C_{\mu}$. The ordering $\leq_{\mu}$ is called the discrete exponential ordering. Note that the restriction $\mu<1$ in [7] is not needed here.

The following result follows immediately from the definition of the ordering $\leq_{\mu}$ (see also [7, Proposition 1]). It gives a necessary and sufficient condition for the map $T$ defined by (1.2) to be monotone. Recall that $T$ is said to be monotone (increasing, order preserving) on $\mathbb{R}^{k+1}$ with respect to $\leq_{\mu}$ if

$$
T(y) \geq_{\mu} T(x) \quad \text { whenever } x, y \in \mathbb{R}^{k+1} \text { satisfy } x \leq_{\mu} y .
$$

Theorem 2.1. Let $\mu \geq 0$. The map $T$ defined by (1.2) is monotone with respect to $\leq_{\mu}$ if and only if

$$
f(y)-f(x) \geq \mu\left(y_{0}-x_{0}\right) \quad \text { whenever } x, y \in \mathbb{R}^{k+1} \text { satisfy } x \leq_{\mu} y .
$$

A relatively easily verifiable sufficient condition for (2.3) to hold is given below.

Proposition 2.2 [7, Proposition 2]. Let $\mu>0$. Condition (2.3) holds if there exist constants $L_{i}, i=0,1, \ldots, k$ such that

$$
f(y)-f(x) \geq \sum_{i=0}^{k} L_{i}\left(y_{i}-x_{i}\right) \quad \text { whenever } x_{i} \leq y_{i} \text { for } i=0,1, \ldots, k
$$

and

$$
\mu+\sum_{i=1}^{k} L_{i}^{-} \mu^{-i} \leq L_{0}
$$

where $L_{i}^{-}=\max \left\{0,-L_{i}\right\}$.

Note that in both previous results the domain $\mathbb{R}^{k+1}$ of $T$ can be replaced with a subset of $\mathbb{R}^{k+1}$.

If $f$ is differentiable, then the constants $L_{i}$ in (2.4) may be viewed as the infima of the partial derivatives $\partial f / \partial x_{i}(x)$, where the infimum is taken over all $x \in \mathbb{R}^{k+1}$.

The next theorem shows that a mild strengthening of the monotonicity condition (2.3) implies that $T$ is eventually strongly monotone.

Theorem 2.3. Let $\mu>0$ and suppose that

$$
f(y)-f(x)>\mu\left(y_{0}-x_{0}\right) \quad \text { whenever } x, y \in \mathbb{R}^{k+1} \text { satisfy } x<_{\mu} y .
$$

Then, $T^{k}$ is strongly monotone with respect to $\leq_{\mu}$, that is,

$$
T^{k}(y) \gg_{\mu} T^{k}(x) \quad \text { whenever } x, y \in \mathbb{R}^{k+1} \text { satisfy } x<_{\mu} y .
$$


Proof. Let $x, y \in \mathbb{R}^{k+1}$ satisfy $x<_{\mu} y$. We must show that $T^{k}(y) \gg_{\mu} T^{k}(x)$. In view of the definition of int $C_{\mu}$ and the relation

$$
T^{k}(x)=\left(f\left(T^{k-1}(x)\right), f\left(T^{k-2}(x)\right), \ldots, f(T(x)), f(x), x_{0}\right), \quad x \in \mathbb{R}^{k+1},
$$

the last inequality is equivalent to the system of inequalities

$$
f(y)-f(x)>\mu\left(y_{0}-x_{0}\right)>0
$$

and

$$
f\left(T^{i+1}(y)\right)-f\left(T^{i+1}(x)\right)>\mu\left(f\left(T^{i}(y)\right)-f\left(T^{i}(x)\right)\right)>0
$$

for $i=0,1, \ldots, k-2$. Since $x<_{\mu} y$, it follows that $y_{0}-x_{0}>0$. (Otherwise, the condition $y-x \in C_{\mu}$ would imply that $y=x$, a contradiction.) Consequently, (2.6) implies (2.9). Since $T$ is monotone, $T(y) \geq_{\mu} T(x)$. Further, by virtue of (2.9) and the definition of $T$, we have

$$
(T(y))_{0}-(T(x))_{0}=f(y)-f(x)>0
$$

and hence $T(y)>_{\mu} T(x)$. Using (2.6) again, we find

$$
f(T(y))-f(T(x))>\mu(f(y)-f(x))>0 .
$$

Thus, (2.10) holds for $i=0$. Suppose for induction that (2.10) holds for some $i \geq 0$. By monotonicity, $T^{i+2}(y) \geq_{\mu} T^{i+2}(x)$. Moreover, in view of (2.10) and the definition of $T$, we have

$$
\left(T^{i+2}(y)\right)_{0}-\left(T^{i+2}(x)\right)_{0}=f\left(T^{i+1}(y)\right)-f\left(T^{i+1}(x)\right)>0 .
$$

Consequently, $T^{i+2}(y)>_{\mu} T^{i+2}(x)$ and therefore (2.6) and (2.10) imply that

$$
f\left(T^{i+2}(y)\right)-f\left(T^{i+2}(x)\right)>\mu\left(f\left(T^{i+1}(y)\right)-f\left(T^{i+1}(x)\right)\right)>0 .
$$

Thus, (2.10) holds for all $i=0,1,2, \ldots$. As noted before, (2.9) and (2.10) imply that $T^{k}(y) \gg_{\mu} T^{k}(x)$.

The next result is similar to Proposition 2.2. It gives a sufficient condition for assumption (2.6) of Theorem 2.3 to hold.

Proposition 2.4. Let $\mu>0$. Then, (2.6) holds if (2.4) holds and the inequality in (2.5) is strict,

$$
\mu+\sum_{i=1}^{k} L_{i}^{-} \mu^{-i}<L_{0} .
$$

The proof of Proposition 2.4 is an obvious modification of the proof of [7, Proposition 2] and thus it is omitted. 
In the next theorem, we describe some further properties of $T$ under the additional assumption that $f$ is continuous and positively homogeneous on $C_{\mu}$. In particular, it can be used to ensure the existence of a strongly positive eigenvector of $T$.

Theorem 2.5. Suppose that there exists $\mu \geq 0$ such that $f$ is continuous on $C_{\mu}$ and (1.6) and (2.3) hold on $C_{\mu}$. Then, the following hold.

(i) $T$ is a continuous, positively homogeneous, and monotone selfmapping of $C_{\mu}$.

(ii) If, in addition, it is assumed that

$$
f\left(\mu^{k}, \mu^{k-1}, \ldots, 1\right)>\mu^{k+1},
$$

then the characteristic equation (1.8) has a unique root $\lambda_{0}$ in $(\mu, \infty)$. This root $\lambda_{0}$ is an eigenvalue of $T$ and $u_{\lambda_{0}}=\left(\lambda_{0}^{k}, \lambda_{0}^{k-1}, \ldots, 1\right)$ is a corresponding strongly positive eigenvector, that is,

$$
T\left(u_{\lambda_{0}}\right)=\lambda_{0} u_{\lambda_{0}}, \quad u_{\lambda_{0}} \gg_{\mu} 0
$$

(iii) If instead of (2.3) the stronger condition (2.6) is assumed, then (2.16) holds.

Proof. (i) The continuity and the positive homogeneity of $T$ are evident. The monotonicity of $T$ is a consequence of Theorem 2.1. The fact that $T$ maps $C_{\mu}$ into itself follows from the monotonicity of $T$ and the equality $T(0)=0$.

(ii) Define

$$
h(\lambda)=\lambda^{k+1}-f\left(\lambda^{k}, \lambda^{k-1}, \ldots, 1\right), \quad \lambda \geq \mu .
$$

Since $\left(\lambda^{k}, \lambda^{k-1}, \ldots, 1\right) \geq_{\mu}(0,0, \ldots, 0)$ for $\lambda \geq \mu$ and $f$ is continuous on $C_{\mu}, h$ is continuous on $[\mu, \infty)$. Further, by virtue of $(2.16), h(\mu)<0$ and, in view of $(1.6)$, we have

$$
h(\lambda)=\lambda^{k}\left(\lambda-f\left(1, \lambda^{-1}, \ldots, \lambda^{-k}\right)\right) \longrightarrow \infty \quad \text { as } \lambda \longrightarrow \infty .
$$

This implies the existence of $\lambda_{0}>\mu$ such that $h\left(\lambda_{0}\right)=0$. This $\lambda_{0}$ is a root of (1.8) and conclusion (2.17) is an immediate consequence of the definitions of $T$ and the strong ordering $\ll_{\mu}$. It remains to show that $(1.8)$ has no other root in $(\mu, \infty)$. Let $\lambda>\mu$ be a root of (1.8). Define $u_{\lambda}=\left(\lambda^{k}, \lambda^{k-1}, \ldots, 1\right)$. It is easily seen that

$$
T\left(u_{\lambda}\right)=\lambda u_{\lambda}, \quad u_{\lambda} \gg_{\mu} 0
$$

Thus, $u_{\lambda}$ is a strongly positive eigenvector of the continuous, positively homogeneous and monotone selfmapping $T$ of $C_{\mu}$. According to a result of Kloeden and Rubinov [3, Corollary 3.1], the corresponding eigenvalue $\lambda$ coincides with the spectral radius of $T$ and hence it is uniquely determined.

(iii) Clearly, $\left(\mu^{k}, \mu^{k-1}, \ldots, 1\right)>_{\mu}(0,0, \ldots, 0)$. By virtue of $(2.6)$, this together with $f(0$, $0, \ldots, 0)=0$, implies (2.16).

Remark 2.6. The previous proof shows that in case (ii) of Theorem $2.5, \lambda_{0}<1$ if and only if $\mu<1$ and $f(1,1, \ldots, 1)<1$. 
We conclude this section with some corollaries of the previous results for (1.7), a special case of (1.1) when

$$
f\left(x_{0}, x_{1}, \ldots, x_{k}\right)=\sum_{i=0}^{k} K_{i} x_{i}+b \max \left\{x_{0}, x_{1}, \ldots, x_{r}\right\}
$$

As in Section 1, we assume that $k \geq r$ in (1.7).

Corollary 2.7. Suppose that $b \geq 0$ and $\mu>0$. Then, the following hold.

(i) Condition (2.3) holds for (1.7) if (1.12) holds.

(ii) Condition (2.6) holds for (1.7) if (1.12) holds with a strict inequality.

(iii) Condition (2.16) holds for (1.7) if (1.12) and one of the following hold:

(a) $b>0$, or

(b) $b=0$ and $K_{i}>0$ for some $i \in\{1,2, \ldots, k\}$, or

(c) $b=0, K_{i} \leq 0$ for $i=1,2, \ldots, k$ and the inequality in (1.12) is strict.

In that case, (1.14) has a unique root $\lambda_{0}$ in $(\mu, \infty)$. Furthermore, $\lambda_{0}<1$ if and only if $\mu<1$ and (1.20) holds.

Proof. Clearly, for $f$ defined by (2.21), condition (2.4) holds with $L_{i}=K_{i}$ for $i=0,1, \ldots, k$. Consequently, conclusions (i) and (ii) follow immediately from Propositions 2.2 and 2.4. To prove (iii), observe that, in view of (1.12), we have

$$
\begin{aligned}
f\left(\mu^{k}, \mu^{k-1}, \ldots, 1\right) & =\mu^{k}\left(\sum_{i=0}^{k} K_{i} \mu^{-i}+b \max \left\{1, \mu^{-1}, \ldots, \mu^{-r}\right\}\right) \\
& \geq \mu^{k}\left(K_{0}-\sum_{i=1}^{k} K_{i}^{-} \mu^{-i}\right) \geq \mu^{k+1} .
\end{aligned}
$$

If (a), (b), or (c) holds, then one of the above inequalities is strict and thus (2.16) holds. The last two conclusions of (iii) follow from Theorem 2.5(ii) and Remark 2.6.

\section{Main results}

In the theorems below, we assume that $f$ is positively homogeneous and satisfies either the monotonicity condition (2.3) or (2.6). Sufficient conditions for (2.3) and (2.6) to hold were given in Section 2 (see Propositions 2.2 and 2.4). The first theorem gives an upper estimate for the solutions of inequality (1.5).

Theorem 3.1. Suppose that there exists $\mu \geq 0$ such that (1.6) and (2.3) hold. If the characteristic equation (1.8) has a root $\lambda_{0}$ in $(\mu, \infty)$, then for every solution $\left(y_{n}\right)_{n \geq-k}$ of (1.5) there exists a positive constant $M=M\left(y_{0}, y_{-1}, \ldots, y_{-k}\right)$ such that

$$
y_{n} \leq M \lambda_{0}^{n}, \quad n \geq-k .
$$

The existence of a root $\lambda_{0}$ of $(1.8)$ in $(\mu, \infty)$ can be guaranteed by Theorem 2.5(ii). We have the following corollary of Theorems 2.5 and 3.1 . 
Corollary 3.2. Suppose that there exists $\mu \geq 0$ such that $f$ is continuous on $C_{\mu}$ and conditions (1.6), (2.3), and (2.16) hold. Then, (1.8) has a unique root $\lambda_{0}$ in $(\mu, \infty)$ and (3.1) holds for every solution $\left(y_{n}\right)_{n \geq-k}$ of (1.5) with a positive constant $M$ depending on the initial data $\left(y_{0}, y_{-1}, \ldots, y_{-k}\right)$.

Remark 3.3. According to Theorem 2.5(iii), condition (2.16) automatically holds if the monotonicity assumption (2.3) in Corollary 3.2 is replaced with the strong monotonicity condition (2.6).

Remark 3.4. Theorem 1.1 in Section 1 is a consequence of Corollaries 2.7 and 3.2.

Before we present the proof of Theorem 3.1, we establish a comparison theorem which is interesting in its own right. Note that in this theorem we merely assume the monotonicity condition (2.3).

Theorem 3.5. Suppose (2.3) holds for some $\mu \geq 0$. Let $\left(x_{n}\right)_{n \geq-k}$ and $\left(y_{n}\right)_{n \geq-k}$ be solutions of (1.1) and (1.5), respectively, such that

$$
\left(y_{0}, y_{-1}, \ldots, y_{-k}\right) \leq_{\mu}\left(x_{0}, x_{-1}, \ldots, x_{-k}\right) .
$$

Then, for all $n \geq 0$,

$$
\left(y_{n}, y_{n-1}, \ldots, y_{n-k}\right) \leq_{\mu}\left(x_{n}, x_{n-1}, \ldots, x_{n-k}\right) .
$$

In particular,

$$
y_{n} \leq x_{n}, \quad n \geq-k .
$$

Proof. We will prove (3.3) by induction on $n$. By assumption (3.2), (3.3) holds for $n=0$. Suppose for induction that (3.3) holds for some $n \geq 0$. In view of the definition of the ordering $\leq_{\mu}$, (3.3) implies that

$$
x_{i}-y_{i} \geq \mu\left(x_{i-1}-y_{i-1}\right) \geq 0
$$

for $i=n-k+1, n-k+2, \ldots, n$. Using (1.1) and (1.5), we find for $n \geq 0$,

$$
x_{n+1}-y_{n+1} \geq f\left(x_{n}, \ldots, x_{n-k}\right)-f\left(y_{n}, \ldots, y_{n-k}\right) \geq \mu\left(x_{n}-y_{n}\right),
$$

the last inequality being a consequence of (2.3) and (3.3). Thus, (3.5) also holds for $i=$ $n+1$. Therefore,

$$
\left(y_{n+1}, y_{n}, \ldots, y_{n+1-k}\right) \leq_{\mu}\left(x_{n+1}, x_{n}, \ldots, x_{n+1-k}\right) .
$$

Thus, (3.3) is confirmed for all $n \geq 0$. Conclusion (3.4) follows from (3.3) and the definition of $C_{\mu}$.

We are in a position to give a proof of Theorem 3.1.

Proof of Theorem 3.1. Let $\left(y_{n}\right)_{n \geq-k}$ be a solution of (1.5). Consider the solution $\left(x_{n}\right)_{n \geq-k}$ of (1.1) with initial data

$$
\left(x_{0}, x_{-1}, \ldots, x_{-k}\right)=\left(y_{0}, y_{-1}, \ldots, y_{-k}\right)
$$


By Theorem 3.5, $y_{n} \leq x_{n}$ for $n \geq-k$. Therefore, it is enough to show that

$$
x_{n} \leq M \lambda_{0}^{n}, \quad n \geq-k,
$$

for some $M>0$. Since $\lambda_{0}>\mu$, the vector $u_{\lambda_{0}}=\left(1, \lambda_{0}^{-1}, \ldots, \lambda_{0}^{-k}\right)$ is strongly positive, $u_{\lambda_{0}} \gg_{\mu} 0$. Consequently,

$$
\left(x_{0}, x_{-1}, \ldots, x_{-k}\right) \leq_{\mu} M u_{\lambda_{0}}=\left(M, M \lambda_{0}^{-1}, \ldots, M \lambda_{0}^{-k}\right)
$$

for all sufficiently large $M$. Since $\lambda_{0}$ is a root of (1.8) and $f$ is positively homogeneous, $\left(M \lambda_{0}^{n}\right)_{n \geq-k}$ is a solution of (1.1). Estimate (3.9) now follows from (3.10) and Theorem 3.5 applied to the solutions $\left(x_{n}\right)_{n \geq-k}$ and $\left(M \lambda_{0}^{n}\right)_{n \geq-k}$ of $(1.1)$.

Remark 3.6. The constant $M$ in (3.1) of Theorem 3.1 can be computed explicitly from (3.10) (where $x_{i}=y_{i}$ for $i=-k,-k+1, \ldots, 0$ ). Writing the system of inequalities corresponding to (3.10) from the definition of the ordering $\leq_{\mu}$, it can be shown that $M$ in (3.1) can be taken as

$$
M=K \max \left\{\left|y_{0}\right|,\left|y_{-1}\right|, \ldots,\left|y_{-k}\right|\right\}
$$

where $K$ is a positive constant independent of the initial data $\left(y_{0}, y_{-1}, \ldots, y_{-k}\right)$.

Our next aim is to show that for the nontrivial solutions $\left(x_{n}\right)_{n \geq-k}$ of (1.1) starting from $C_{\mu}$, the exponential estimate (3.1) of Theorem 3.1 can be replaced with the more precise limit relation

$$
\lim _{n \rightarrow \infty}\left(\lambda_{0}^{-n} x_{n}\right)=L
$$

where $L$ is a positive constant depending on the initial data.

Theorem 3.7. Suppose that there exists $\mu>0$ such that $f$ is continuous on $C_{\mu}$ and (1.6) and (2.6) hold. Then, for every solution $\left(x_{n}\right)_{n \geq-k}$ of (1.1) with initial data $\left(x_{0}, x_{-1}, \ldots, x_{-k}\right) \in$ $C_{\mu} \backslash\{0\}$, there exists a positive constant $L=L\left(x_{0}, x_{-1}, \ldots, x_{-k}\right)$ such that (3.12) holds, where $\lambda_{0}$ is the unique root of $(1.8)$ in $(\mu, \infty)$.

Note that if $f$ in Theorem 3.7 is linear, then the value of the limit (3.12) can be given explicitly in terms of the initial data $\left(x_{0}, x_{-1}, \ldots, x_{-k}\right)$ (see [2] or [4] for details).

The proof of Theorem 3.7 will be based on a nonlinear version of the Perron-Frobenius theorem due to Kloeden and Rubinov [3] adapted to our situation. For further related results, see [5].

Theorem 3.8. Let $\mu \geq 0$. Suppose that $T: C_{\mu} \rightarrow \mathbb{R}^{k+1}$ is a continuous, positively homogeneous map with the following properties:

(i) $T\left(C_{\mu}\right) \subset C_{\mu}$,

(ii) there exist $\lambda>0$ and $u \gg_{\mu} 0$ such that $T(u)=\lambda u$,

(iii) $T$ is monotone on $C_{\mu}$, that is,

$$
T(y) \geq_{\mu} T(x) \quad \text { whenever } x, y \in C_{\mu} \text { satisfy } x \leq_{\mu} y,
$$


(iv) some iterate $T^{s}(s \geq 1)$ of $T$ is strongly monotone on $C_{\mu}$, that is,

$$
T^{s}(y) \gg_{\mu} T^{s}(x) \quad \text { whenever } x, y \in C_{\mu} \text { satisfy } x<_{\mu} y
$$

Then, for every $x \in C_{\mu} \backslash\{0\}$, there exists a positive constant $K=K(x)$ such that

$$
\lambda^{-n} T^{n}(x) \longrightarrow K u \quad \text { as } n \longrightarrow \infty .
$$

Theorem 3.8 is a consequence of [3, Corollary 5.2 and Remark 5.1] applied to the scaled map $\widetilde{T}=\lambda^{-1} T$.

Proof of Theorem 3.7. We will prove Theorem 3.7 by applying Theorem 3.8 to the map $T$ defined by (1.2). Theorems 2.3 and 2.5 show that the hypotheses of Theorem 3.8 hold with $\lambda=\lambda_{0}$ and $u=\left(\lambda_{0}^{k}, \lambda_{0}^{k-1}, \ldots, 1\right)$, where $\lambda_{0}$ is the unique root of $(1.8)$ in $(\mu, \infty)$. By the application of Theorem 3.8, we conclude that if $\left(x_{0}, x_{-1}, \ldots, x_{-k}\right) \in C_{\mu} \backslash\{0\}$, then

$$
\lambda_{0}^{-n} T^{n}\left(x_{0}, x_{-1}, \ldots, x_{-k}\right) \longrightarrow K\left(\lambda_{0}^{k}, \lambda_{0}^{k-1}, \ldots, 1\right) \quad \text { as } n \longrightarrow \infty
$$

for some $K>0$. By virtue of (1.3), the last limit relation is equivalent to (3.12) with $L=$ $K \lambda_{0}^{k}$.

Remark 3.9. Theorem 1.2 in Section 1 is a consequence of Theorem 3.7 and Corollary 2.7.

Now, we present a theorem concerning the behavior of the solutions of (1.10). We will assume that the linear part of (1.10) generates a monotone system with respect to the ordering $\leq_{\mu}$ and we use the variation-of-constants formula to obtain an exponential estimate for the growth of the solutions. As in Section 1, we assume that $k \geq r$ in (1.10).

Theorem 3.10. Suppose that there exist $\mu>0$ and a function $h: \mathbb{R}_{+}^{r+1} \rightarrow \mathbb{R}_{+}$such that for $n \geq 0$ and $x, y \in \mathbb{R}^{r+1}$,

$$
\begin{gathered}
\left|g\left(n, x_{0}, x_{1}, \ldots, x_{r}\right)\right| \leq h\left(\left|x_{0}\right|,\left|x_{1}\right|, \ldots,\left|x_{r}\right|\right), \\
h(y) \geq h(x) \quad \text { whenever } 0 \leq x_{i} \leq y_{i} \text { for } i=0,1, \ldots, r, \\
h \text { is continuous and positively homogeneous on } C_{\mu}, \\
\mu+\sum_{i=1}^{k} K_{i}^{-} \mu^{-i} \leq K_{0}, \quad K_{i}^{-}=\max \left\{0,-K_{i}\right\}
\end{gathered}
$$

and one of the following holds:

(a) $h\left(\mu^{r}, \mu^{r-1}, \ldots, 1\right)>0$, or

(b) $h\left(\mu^{r}, \mu^{r-1}, \ldots, 1\right)=0$ and $K_{i}>0$ for some $i \in\{1,2, \ldots, k\}$, or

(c) $h\left(\mu^{r}, \mu^{r-1}, \ldots, 1\right)=0, K_{i} \leq 0$ for $i=1,2, \ldots, k$ and the inequality in (3.20) is strict.

Then, for every solution $\left(x_{n}\right)_{n \geq-k}$ of (1.10) there exists a positive constant $M=M\left(x_{0}\right.$, $\left.x_{-1}, \ldots, x_{-k}\right)$ such that

$$
\left|x_{n}\right| \leq M \lambda_{0}^{n}, \quad n \geq-k
$$


where $\lambda_{0}$ is the unique root of the equation

$$
\lambda^{k+1}=\sum_{i=0}^{k} K_{i} \lambda^{k-i}+h\left(\lambda^{k}, \lambda^{k-1}, \ldots, \lambda^{k-r}\right)
$$

in the interval $(\mu, \infty)$.

Proof. First, we show that (3.22) has a unique root in $(\mu, \infty)$. We will apply Theorem 2.5(ii) to the equation

$$
x_{n+1}=\sum_{i=0}^{k} K_{i} x_{n-i}+h\left(x_{n}, x_{n-1}, \ldots, x_{n-r}\right), \quad n \geq 0 .
$$

Equation (3.23) is a special case of (1.1) when

$$
f\left(x_{0}, x_{1}, \ldots, x_{k}\right)=\sum_{i=0}^{k} K_{i} x_{i}+h\left(x_{0}, x_{1}, \ldots, x_{r}\right) .
$$

Conditions (3.18) and (3.20) imply that assumptions (2.4) and (2.5) of Proposition 2.2 hold for (3.23) on $C_{\mu}$ with $L_{i}=K_{i}$ for $i=0,1, \ldots, k$. By Proposition 2.2, the monotonicity condition (2.3) holds for (3.23) on $C_{\mu}$. By virtue of (3.19), $f$ is continuous and positively homogeneous on $C_{\mu}$. Further, by virtue of (3.19) and (3.20), we have

$$
\begin{aligned}
f\left(\mu^{k}, \mu^{k-1}, \ldots, 1\right) & =\mu^{k}\left(\sum_{i=0}^{k} K_{i} \mu^{-i}+\mu^{-r} h\left(\mu^{r}, \mu^{r-1}, \ldots, 1\right)\right) \\
& \geq \mu^{k}\left(K_{0}-\sum_{i=1}^{k} K_{i}^{-} \mu^{-i}\right) \geq \mu^{k+1} .
\end{aligned}
$$

Since any of the conditions (a), (b), or (c) implies that one of the last two inequalities is strict, (2.16) holds. The existence and uniqueness of $\lambda_{0}$ now follows from Theorem 2.5(ii).

Now, we prove (3.21). Let $\left(x_{n}\right)_{n \geq-k}$ be an arbitrary solution of (1.10). Consider the solution $\left(y_{n}\right)_{n \geq-k}$ of the linear equation $(1.11)$ with the same initial data, $\left(y_{0}, y_{-1}, \ldots, y_{-k}\right)=$ $\left(x_{0}, x_{-1}, \ldots, x_{-k}\right)$. Since $\lambda_{0}>\mu$, we have

$$
\left(1, \lambda_{0}^{-1}, \ldots, \lambda_{0}^{-k}\right) \gg_{\mu}(0,0, \ldots, 0) .
$$

Consequently,

$$
\left(y_{0}, y_{-1}, \ldots, y_{-k}\right) \leq_{\mu} M_{1}\left(1, \lambda_{0}^{-1}, \ldots, \lambda_{0}^{-k}\right)
$$

for all sufficiently large $M_{1}>0$. By Proposition 2.2, (3.20) implies that the monotonicity condition (2.3) holds for the linear equation (1.11). Therefore, we can apply Theorem 3.5 to (1.11) and from (3.27) we obtain

$$
y_{n} \leq M_{1} w_{n}, \quad n \geq-k
$$


where $\left(w_{n}\right)_{n \geq-k}$ is the solution of $(1.11)$ with initial data $\left(w_{0}, w_{-1}, \ldots, w_{-k}\right)=\left(1, \lambda_{0}^{-1}, \ldots\right.$, $\left.\lambda_{0}^{-k}\right)$. The same argument applied to the solution $\left(-y_{n}\right)_{n \geq-k}$ of (1.11) yields the existence of $M_{2}>0$ such that

$$
-y_{n} \leq M_{2} w_{n}, \quad n \geq-k
$$

Consequently,

$$
\left|y_{n}\right| \leq M_{3} w_{n}, \quad n \geq-k
$$

where $M_{3}=\max \left\{M_{1}, M_{2}\right\}$. Here, we have used the fact that $w_{n} \geq 0$ for $n \geq-k$ which follows from Theorem 3.5 and (3.26). We will show that (3.21) holds with

$$
M=\max \left\{M_{3},\left|x_{0}\right|,\left|x_{-1}\right| \lambda_{0},\left|x_{-2}\right| \lambda_{0}^{2}, \ldots,\left|x_{-k}\right| \lambda_{0}^{k}\right\}
$$

By the definition of $M$, we have

$$
\left|x_{i}\right| \leq M \lambda_{0}^{i} \quad \text { for } i=-k,-k+1, \ldots, 0
$$

Suppose that $n \geq 1$ and

$$
\left|x_{i}\right| \leq M \lambda_{0}^{i} \quad \text { for } i=-k,-k+1, \ldots, n-1 \text {. }
$$

By the induction principle, the proof will be complete if we show that (3.33) also holds for $i=n$. By the variation-of-constants formula (see [11, Lemma 1]), the solution $x_{n}$ of (1.10) can be written in the form

$$
x_{n}=y_{n}+\sum_{i=0}^{n-1} v_{n-i-1} g\left(i, x_{i}, x_{i-1}, \ldots, x_{i-r}\right), \quad n \geq 0,
$$

where $y_{n}$ has the meaning as before and $\left(v_{n}\right)_{n \geq-k}$ is the (fundamental) solution of the linear equation $(1.11)$ with initial data $\left(v_{0}, v_{-1}, \ldots, v_{-k}\right)=(1,0, \ldots, 0)$. Since $(1,0, \ldots, 0) \geq_{\mu}$ $(0,0, \ldots, 0)$, Theorem 3.5 implies that $v_{n} \geq 0$ for $n \geq 0$. Using (3.17), (3.18), (3.30), and (3.33) in (3.34), we find

$$
\left|x_{n}\right| \leq M w_{n}+\sum_{i=0}^{n-1} v_{n-i-1} h\left(M \lambda_{0}^{i}, M \lambda_{0}^{i-1}, \ldots, M \lambda_{0}^{i-r}\right)
$$

Writing the variation-of-constants formula for the solution $\left(\lambda_{0}^{n}\right)_{n \geq-k}$ of (3.23), we obtain for $n \geq 0$,

$$
\lambda_{0}^{n}=w_{n}+\sum_{i=0}^{n-1} v_{n-i-1} h\left(\lambda_{0}^{i}, \lambda_{0}^{i-1}, \ldots, \lambda_{0}^{i-r}\right)
$$


where $w_{n}$ and $v_{n}$ are the solutions of (1.11) defined as before. This and the positive homogeneity of $h$ imply that the right-hand side of (3.35) is equal to $M \lambda_{0}^{n}$. Thus, we have shown that (3.33) implies that $\left|x_{n}\right| \leq M \lambda_{0}^{n}$.

The same argument as in Remark 2.6 shows that the constants $M_{1}$ and $M_{2}$ in the previous proof and hence $M$ in (3.21) can be written in the form (3.11) (with $y$ replaced with $x$ ). Consequently, Theorem 3.10 combined with Remark 2.6 yields the following stability criterion.

Theorem 3.11. In addition to the hypotheses of Theorem 3.10, suppose that $\mu<1$ and

$$
\sum_{i=0}^{k} K_{i}+h(1,1, \ldots, 1)<1 .
$$

Then, the zero solution of (1.10) is globally exponentially stable.

Remark 3.12. Theorems 1.3 and 1.4 in Section 1 follow from Theorems 3.10 and 3.11, respectively, when $h\left(x_{0}, x_{1}, \ldots, x_{r}\right)=b \max \left\{x_{0}, x_{1}, \ldots, x_{r}\right\}$.

\section{Acknowledgments}

The first author was partially supported by the M.C.T. (Spain) and FEDER under the project BFM 2001-3884-C02-02. The second author was partially supported by the Hungarian National Foundation for Scientific Research (OTKA) Grant no. T 046929.

\section{References}

[1] C. T. H. Baker and A. Tang, Generalized Halanay inequalities for Volterra functional differential equations and discretized versions, Volterra Equations and Applications (Arlington, Tex, 1996), Stability Control Theory Methods Appl., vol. 10, Gordon and Breach, Amsterdam, 2000, pp. 39-55.

[2] R. D. Driver, G. Ladas, and P. N. Vlahos, Asymptotic behavior of a linear delay difference equation, Proc. Amer. Math. Soc. 115 (1992), no. 1, 105-112.

[3] P. E. Kloeden and A. M. Rubinov, A generalization of the Perron-Frobenius theorem, Nonlinear Anal. Ser. A: Theory Methods 41 (2000), no. 1-2, 97-115.

[4] I.-G. E. Kordonis and Ch. G. Philos, On the behavior of the solutions for linear autonomous neutral delay difference equations, J. Differ. Equations Appl. 5 (1999), no. 3, 219-233.

[5] U. Krause, Relative stability for ascending and positively homogeneous operators on Banach spaces, J. Math. Anal. Appl. 188 (1994), no. 1, 182-202.

[6] - The asymptotic behavior of monotone difference equations of higher order, Comput. Math. Appl. 42 (2001), no. 3-5, 647-654.

[7] U. Krause and M. Pituk, Boundedness and stability for higher order difference equations, J. Difference Equ. Appl. 10 (2004), no. 4, 343-356.

[8] E. Liz and J. B. Ferreiro, A note on the global stability of generalized difference equations, Appl. Math. Lett. 15 (2002), no. 6, 655-659.

[9] E. Liz, A. Ivanov, and J. B. Ferreiro, Discrete Halanay-type inequalities and applications, Nonlinear Anal. 55 (2003), no. 6, 669-678.

[10] S. Mohamad and K. Gopalsamy, Continuous and discrete Halanay-type inequalities, Bull. Austral. Math. Soc. 61 (2000), no. 3, 371-385. 
[11] M. Pituk, Global asymptotic stability in a perturbed higher-order linear difference equation, Comput. Math. Appl. 45 (2003), no. 6-9, 1195-1202.

[12] H. L. Smith, Monotone Dynamical Systems. An Introduction to the Theory of Competitive and Cooperative Systems, Mathematical Surveys and Monographs, vol. 41, American Mathematical Society, Rhode Island, 1995.

[13] H. L. Smith and H. R. Thieme, Monotone semiflows in scalar non-quasi-monotone functionaldifferential equations, J. Math. Anal. Appl. 150 (1990), no. 2, 289-306.

Eduardo Liz: Departamento de Matemática Aplicada II, ETSI Telecomunicación, Universidade de Vigo, Campus Marcosende, 36280 Vigo, Spain

E-mail address: eliz@dma.uvigo.es

Mihály Pituk: Department of Mathematics and Computing, University of Veszprem, P.O. Box 158, 8201 Veszprem, Hungary

E-mail address: pitukm@almos.vein.hu 